\title{
Numerical Solution of Singular ODE Eigenvalue Problems in Electronic Structure Computations
}

\author{
Robert Hammerling \\ Vienna University of Technology \\ Center for Computational Materials Science \\ Gußhausstraße 25-25a, A-1040 Wien, AUSTRIA \\ rh@cms.tuwien.ac.at
}

This work was supported by the City of Vienna Science and Technology Fund

via the project 'Mathematik und ... 2007'.

\author{
Othmar Koch, Christa Simon, Ewa B. Weinmüller \\ e.weinmueller@tuwien.ac.at \\ http://www.math.tuwien.ac.at/〜ewa/ \\ Vienna University of Technology \\ Institute for Analysis and Scientific Computing \\ Wiedner Hauptstraße 8-10, A-1040 Wien, AUSTRIA
}

February 24, 2010

\begin{abstract}
We put forward a new method for the solution of eigenvalue problems for (systems of) ordinary differential equations, where our main focus is on eigenvalue problems for singular Schrödinger equations arising for example in electronic structure computations. In most established standard methods, the generation of the starting values for the computation of eigenvalues of higher index is a critical issue. Our approach comprises two stages: First we generate rough approximations by a matrix method, which yields several eigenvalues and associated eigenfunctions simultaneously, albeit with moderate accuracy. In a second stage, these approximations are used as starting values for a collocation method which yields approximations of high accuracy efficiently due to an adaptive mesh selection strategy, and additionally provides reliable error estimates. We successfully apply our method to the solution of the quantum mechanical Kepler, Yukawa and the coupled ODE Stark problems.
\end{abstract}

keywords: electronic structure computation, polynomial collocation, fullpotential core solver, singular eigenvalue problems

MSC 65L15, 65L60, 65N25, 81-08 PACS 31.15ae, 31.15es, 32.60.+i 


\section{Introduction}

The effective one-particle model of electronic structure theory of non-relativistic, quantum $N$-body problem comprises the determination of the $N$ lowest eigenvalues and corresponding eigenfunctions of a one-body Schrödinger operator. The partial differential operator with effective local (multiplicative) potential

$$
\begin{aligned}
& \mathcal{D} \subset L^{2}\left(\mathbb{R}^{3}, \mathbb{C}\right) \rightarrow L^{2}\left(\mathbb{R}^{3}, \mathbb{C}\right) \\
& \psi \mapsto\left(-\frac{1}{2} \triangle+V(x)\right) \psi,
\end{aligned}
$$

arises in Hartree as well as density functional theory (DFT). The potential itself is determined in a nonlinear way from the eigenorbitals, but assumed already given in this paper. For all atomic problems in the central field approximation and the core states in molecular and solid-state calculations, a spherical potential is assumed and the PDE operator reduces to an ODE operator on $L^{2}\left(\mathbb{R}^{+}, \mathbb{C}\right)$. A small perturbation from a spherical potential can be described by a coupled set of ODEs on $L^{2}\left(\mathbb{R}^{+}, \mathbb{C}^{M}\right)$, where $M=\left(l_{\max }+1\right)^{2}$ is determined by the angular momentum cut-off $[10,32]$. The approximate, numerical computation of the ODE-EVP has been studied since the time of D. Hartree and J. Slater [11, 30].

Although it is not the major source of computation time in solid-state applications, all electronic structure codes contain some form of core-state solver [8, 9] as an essential ingredient [23]. Alternative methods exist [29] and important mathematical questions still remain open. For the formulation of the computational problem, the following important questions need to be addressed: Since long range potentials on unbounded intervals lead to an infinite point spectrum, how many of these eigenvalues are of interest? Should the problem solution be attempted on the interval $r \in(0, \infty)$ or on a suitably truncated interval $\left(0, r_{\max }\right]$, where $r_{\max }$ is chosen appropriately? How are problems treated where the potential is not given in a closed form? Note that for the purpose of this paper, we assume that $V$ can be evaluated at arbitrary points. These questions and the resulting solution method are normally only vaguely stated in the physics literature, since the answers are determined by the context, assumed to be understood well-enough by physicists. The standard core state solvers in solid-state electronic structure codes truncate to a bounded interval and use a two-sided shooting method based on the solution of a nonstandard ODE-IVP for $r>0$ to the right,

$$
\begin{aligned}
& L y=\lambda y, \\
& y(r) \sim r^{l+1}, \quad \text { for } r \rightarrow 0,
\end{aligned}
$$

and the corresponding problem starting at $r=r_{\infty} \gg 1$ with integration to the left. Note that the employed multistep method uses the starting values $y(0)$ and $y(h)$, which does not correspond to a Cauchy problem with $y(0), y^{\prime}(0)$ given. The matching is normally done at the outermost inflection point and the eigenparameter $\lambda$ is adapted until a $C^{1}$ function is 
obtained [7].

The actual implementation of the core state solver is further complicated by the fact that relativistic effects are included to some extent, and often in fact the radial Dirac equation is solved instead of the Schrödinger equation. We present our method in this paper from the point of view of numerical analysis with a focus on general methods for singular ODE-EVP and comparison with methods specially adapted to the linear Schrödinger equation.

The outline of the paper is the following: In Section 1 we give a brief review of the theory and an overview of alternative approaches, in Sections 2 and 3 we present the collocation and matrix methods, the two central parts of our computational method. In Section 4 we apply our method to the standard problems of the hydrogen and the Yukawa problem and an ODE system resulting from the dc-Stark effect. The latter is validated against results based on perturbation theory. The direct treatment of the Stark effect by a coupled ODE system is new to the best of our knowledge and of at least didactical value. Finally, Section 5 presents our conclusions.

\section{Eigenvalue Problems for Singular ODEs}

We study eigenvalue problems for systems of linear ordinary differential equations

$$
\begin{aligned}
& L y=\lambda y, \\
& B_{a} y(a)+B_{b} y(b)=0,
\end{aligned}
$$

where $L$ is a linear differential operator of order one or order two which may be singular, particularly we allow $a=-\infty$ and/or $b=\infty$. The application that we focus on is the radial Schrödinger equation

$$
\begin{aligned}
& L y(r):=\left(-\frac{1}{2} \frac{d^{2}}{d r^{2}}+\frac{l(l+1)}{2 r^{2}}+V(r)\right) y(r)=\lambda y(r), \quad r \in(0, \infty), \\
& y(0)=y(\infty)=0
\end{aligned}
$$

where $l \in \mathbb{N}_{0}$. This represents a singular eigenvalue problem [25]. Since the differential operator $L$ is self-adjoint, the spectrum is real [31]. Our aim is to compute the point spectrum of $L$ numerically. Our approach uses first a matrix method, which replaces the continuous problem by a finite discretization whose eigenvalues converge to those of the original problem. This provides crude approximations of a large number of eigenvalues and eigenfunctions simultaneously, which serve as starting values for a solution based on collocation on adaptive meshes with reliable error control.

Alternative to our approach, there is a number of successful codes for the solution of (singular) Sturm-Liouville problems: The code SLEIGN2 [3] uses the so-called Prüfer transformation to transform the linear eigenvalue problem to a nonlinear eigenvalue problem 
believed to be suitable for numerical integration. This task is realized by a two-sided shooting procedure which is iteratively refined until a matching condition at some suitable interior point is satisfied up to a tolerance. Intricate preprocessing is used to prepare for the numerical integration of the initial value problems which is then realized by an explicit Runge-Kutta method.

SLEDGE [24] replaces the original equation by an approximating problem based on step functions which has known exact solutions. Finally, either a one-sided or two-sided shooting method is applied to determine the eigenvalue.

SL02F [22] approximates the given problem by replacing the coefficient functions with piecewise constant approximations.

MATSLISE and MATSCS [20, 21] also use approximation of the coefficient functions and a shooting procedure. This implies that the mesh which is used for the numerical calculations is fixed during the whole shooting procedure.

MATSLise does not offer any possibilities to solve eigenvalue problems for systems of ODEs and also only a limited class of singular problems is covered. MATsCs on the other hand is a code specifically designed for the the solution of eigenvalue problems for systems of coupled, regular equations. All these codes have in common that intricate procedures are integrated to enable computation of particular eigenvalues and eigenfunctions of higher index.

\section{Collocation Method}

In the approach put forward and analyzed in [1], we rewrite the problem (5)-(6) by introducing the following auxiliary quantities: We formally interpret $\lambda$ as a function of $t$ and add the auxiliary differential equation

$$
\lambda^{\prime}(t)=0,
$$

and define

$$
x(t):=\int_{a}^{t}|y(\tau)|^{2} d \tau
$$

whence we have a further differential equation involving a quadratic nonlinearity, and two additional boundary conditions:

$$
x^{\prime}(t)=|y(t)|^{2}, \quad x(a)=0, \quad x(b)=1 .
$$

The resulting augmented system is a boundary value problem in standard form (not an eigenvalue problem) for the set of unknowns $y(t), \lambda(t)$ and $x(t)$ without any further unknown parameters. This system is subsequently solved by polynomial collocation. In this way, at some extra cost we can make use of the elaborate theory and practical usefulness of these methods, particularly for singular problems, and use a code developed by the authors featuring asymptotically correct error estimation and adaptive mesh selection for an efficient and reliable solution of the problem [17]. To treat the unbounded interval, we use an approach successfully applied in $[5,6,16]$ : We split the interval at the point $t=1$ and 
transform the problem posed on $[1, \infty)$ by $t \mapsto 1 / t$. This yields a system of doubled size on the finite interval $(0,1]$, where the differential operator now has an essential singularity at zero. This problem class is in the scope of our numerical approach [2]. Finally, the auxiliary equations (9) and (11) are added.

As the problem becomes nonlinear after the reformulation based on (9)-(11), the solution is sensitive to the starting values for the solution of the associated discrete nonlinear algebraic system. Particularly, for many problems (7), the eigenvalues accumulate at some finite number, which means that for the computation of eigenvalues of higher index, very accurate starting guesses are required. We propose to provide these by the matrix method introduced in the next section.

\section{Matrix Method}

In this approach $[18,25]$, the continuous problem $(5)-(6)$ is replaced by an algebraic eigenvalue problem, whose eigenvalues and eigenvectors are approximations to the eigenvalues and eigenfunctions of the original problem. This can be done by any suitable discretization scheme. For simplicity, we describe the approach for first order problems, but for efficiency we have also implemented a separate module for the direct solution of second order problems. We describe the (singular) problem that results after transformation to a finite interval, see Section 2. We first consider a linear first order eigenvalue problem

$$
\begin{aligned}
& L z(t)=z^{\prime}(t)-\frac{M(t)}{t^{\alpha}} z(t)=\lambda G(t) z(t), \quad t \in(0,1], \alpha \geq 1, \\
& B_{0} z(0)+B_{1} z(1)=0 .
\end{aligned}
$$

This general formulation also includes the case when eigenvalue problems for higher order equations are transformed to a first order system, and also the case of problems on unbounded intervals after transformation to the finite interval $(0,1]$, see [2]. The differential problem (12)-(13) is discretized by any suitable discretization scheme [18]. Since our main interest is in singular problems, we choose the box scheme, which is known to have beneficial convergence properties for this problem class $[12,14]$. The eigenvalues of the resulting generalized algebraic eigenvalue problem

$$
A v=\lambda B v
$$

can be shown to converge to the eigenvalues of the continuous problem $[12,18]$ as the discretization parameter $h$ tends to zero. For many problems in our scope, the matrix $B$ in (14) is singular, whence standard convergence theory for this approach cannot be applied. According to the results in [12], $B$ may be any square matrix. However, standard solution routines for the algebraic eigenvalue problem are commonly designed for the case where $A$ is symmetric and $B$ is symmetric and positive definite. In detail, the approximation scheme 
for the singular first order problem is given by

$$
\begin{aligned}
& \frac{z_{j+1}-z_{j}}{h_{j}}-\frac{1}{2} \frac{M\left(t_{j+1 / 2}\right)}{t_{j+1 / 2}^{\alpha}}\left(z_{j+1}+z_{j}\right)=\lambda \frac{1}{2} G\left(t_{j+1 / 2}\right)\left(z_{j+1}+z_{j}\right), \\
& B_{0} z_{0}+B_{1} z_{N}=0 .
\end{aligned}
$$

Since this numerical scheme uses no evaluation at $t_{0}=0$, it can be applied straight forwardly to singular problems. The resulting matrix eigenvalue problem is given by

$$
\begin{gathered}
\left(\begin{array}{cccccc}
D_{0} & R_{0} & & & & \\
& D_{1} & R_{1} & & & \\
& & \ddots & \ddots & & \\
& & & & D_{N-1} & R_{N-1} \\
B_{0} & & & & & B_{1}
\end{array}\right)\left(\begin{array}{c}
z_{0} \\
z_{1} \\
\vdots \\
z_{N}
\end{array}\right)= \\
\lambda\left(\begin{array}{cccccc}
G_{0} & G_{0} & & & & \\
& G_{1} & G_{1} & & & \\
& & \ddots & \ddots & & \\
& & & & G_{N-1} & G_{N-1} \\
0 & & & & & 0
\end{array}\right)\left(\begin{array}{c}
z_{0} \\
z_{1} \\
\vdots \\
z_{N}
\end{array}\right),
\end{gathered}
$$

where $D_{j}, G_{j}$ and $R_{j}$ are $n \times n$ matrices given by

$$
\begin{aligned}
D_{j} & =-\frac{1}{h_{j}} I-\frac{1}{2}\left(\frac{M\left(t_{j+1 / 2}\right)}{t_{j+1 / 2}^{\alpha}}\right), \\
R_{j} & \left.=\frac{1}{h_{j}} I-\frac{1}{2}\left(\frac{M\left(t_{j+1 / 2}\right)}{t_{j+1 / 2}^{\alpha}}\right)\right), \\
G_{j} & =\frac{1}{2} G\left(t_{j+1 / 2}\right), \quad j=0, \ldots, N-1 .
\end{aligned}
$$

Here $h_{j}$ denotes the (not necessarily equidistant) step width and $I$ is the identity matrix. Since we approximate the solution of (12)-(13) by solving the numerical scheme (15) for a small step size $h_{j}$, our aim is to estimate the approximation quality. Therefore, we are interested in how fast the computed value $\lambda_{n, h}$ converges to the exact eigenvalue $\lambda_{n}^{\text {exact }}$ as $h=\max _{j} h_{j}$ tends to 0 . In [12] the following convergence result is given:

Theorem 3.1 Let $\alpha=1$ in (12)-(13), and assume that if $M(0)$ has eigenvalues with positive real part $\sigma_{0}$, then $\sigma_{0}>2$. If $M \in C^{3}, G \in C^{2}$, then

$$
\max _{1 \leq k \leq \tau}\left|\lambda_{n}^{\text {exact }}-\lambda_{n, h}^{k}\right| \leq C \cdot\left(h^{2}|\ln (h)|^{d-1}\right)^{\frac{1}{\kappa}},
$$

where $\tau$ is the dimension of the eigenspace corresponding to the exact eigenvalue $\lambda_{n}^{\text {exact }}, d$ is the dimension of the largest Jordan box associated with the eigenvalue 0 of $M$, $\kappa$ is the smallest integer $l$ for which the nullspaces $\left(I-\lambda_{n}^{\text {exact }} L^{-1} G\right)^{l}$ and $\left(I-\lambda_{n}^{\text {exact }} L^{-1} G\right)^{l+1}$ coincide and $\lambda_{n, h}^{k}$ are the numerical approximations of $\lambda_{n}^{\text {exact }}$. 
Remark: For the constant $C$ in (18), it turns out that $C=C(n)$, i. e. the approximation quality deteriorates for eigenvalues with higher index, see also [20, 28]. While this can be shown theoretically when the matrix $B$ in (14) is symmetric positive definite [15], for our more general case we only have numerical evidence of this property [28].

\section{Applications}

In this section, we give several numerical examples to illustrate the performance of our approximation method. First, we numerically illustrate our claims on the convergence of our methods. We consider the eigenvalue problem for the hydrogen atom [31]

$$
\begin{aligned}
& -\frac{1}{2} y^{\prime \prime}(r)+\left(\frac{l(l+1)}{2 r^{2}}-\frac{\gamma}{r}\right) y(r)=\lambda y(r), \quad r \in(0, \infty), \\
& y(0)=0, \quad y(\infty)=0
\end{aligned}
$$

where $\gamma=1$ is the nuclear charge. The eigenvalues are known analytically to be equal to $\lambda_{n}=-\frac{1}{2 n^{2}}$. In the following, $n$ denotes the principal quantum number with $n \in \mathbb{N}$. After our transformations to a first order problem on a finite interval, the matrix method from Section 3 converges of second order: Table 1 gives the empirical convergence order $p$ for the approximation of the eigenvalue for $n=4$ and $l=3$ : The solution by collocation

\begin{tabular}{|c|c|c|}
\hline$h$ & $\left|\lambda_{h}-\lambda_{h / 2}\right|$ & $p$ \\
\hline $1 / 50$ & $1.221113 \mathrm{e}-03$ & 2.383187 \\
$1 / 100$ & $2.340697 \mathrm{e}-04$ & 2.121570 \\
$1 / 200$ & $5.378845 \mathrm{e}-05$ & 2.021840 \\
$1 / 400$ & $1.324508 \mathrm{e}-05$ & - \\
\hline
\end{tabular}

Table 1: Convergence order for $\lambda=-\frac{1}{32}$ for example (19)-(20).

methods has also been demonstrated to display the optimal convergence order for singular problems $[12,13,14]$ when applied to the solution of eigenvalue problems as described in Section 2 , see $[1,28]$. In Table 2 we demonstrate the advantage of using adaptive mesh refinement over fixed (equidistant) grids. We show the reduction in the number of grid points which are necessary to achieve the same accuracy on an adaptive mesh as compared to an equidistant mesh. We conclude that our adaptive approach represents a major advantage over the solution on a fixed grid. The observations that the possibility to use the full functionality of our adaptive collocation code for singular boundary value problems [17] yields a significant gain in efficiency, while the required starting values can be cheaply provided with sufficient accuracy by a matrix method, lead us to put forward the resulting combined approach, which we will subsequently test on several examples of physical interest. 


\begin{tabular}{|c|c|c|c|c|}
\hline$\lambda_{n}^{\text {exact }}$ & abs. err. (equidistant) & $N$ & abs. err (adaptive) & $N_{\text {adaptive }}$ \\
\hline$-\frac{1}{18}$ & $1.2040 \mathrm{e}-10$ & 535 & $1.2103 \mathrm{e}-10$ & 240 \\
$-\frac{1}{32}$ & $4.7800 \mathrm{e}-10$ & 488 & $4.7817 \mathrm{e}-10$ & 397 \\
$-\frac{1}{50}$ & $1.6866 \mathrm{e}-09$ & 750 & $1.6944 \mathrm{e}-09$ & 469 \\
\hline
\end{tabular}

Table 2: Example (19)-(20) with $l=2$ : Eigenvalues and errors computed on equidistant meshes as compared to adaptive meshes.

As an example of interest in the physics literature we first discuss the Schrödinger equation for the radially symmetric Yukawa potential,

$$
\begin{aligned}
& -\frac{1}{2} y^{\prime \prime}(r)+\left(\frac{l(l+1)}{2 r^{2}}+V(r)\right) y(r)=\lambda y(r), \quad r \in(0, \infty), \quad V(r)=-\frac{\mathrm{e}^{-\alpha r}}{r}, \\
& y(0)=0, y(\infty)=0
\end{aligned}
$$

where the parameter $\alpha>0$ is called screening parameter. Bound states exist only for values of $\alpha$ below a threshold $\alpha_{c}$. The number of elements in the point spectrum varies with $\alpha$.

Table 3 shows the results of our hybrid method and compares them to values reported in [26]. The most remarkable advantage of our approach is the high accuracy which is confirmed by a reliable a posteriori error estimate. In the adaptive collocation method, 8 collocation points are used. For further comparisons see [28].

\begin{tabular}{|c|c|c|r|r|r|c|c|}
\hline$\alpha$ & $n$ & $l$ & \multicolumn{1}{|c}{$\lambda^{(0)}(\mathrm{MM})$} & \multicolumn{1}{c|}{$N$} & bvpsuite & errest $_{\lambda}$ & {$[26]$} \\
\hline 0.1 & 1 & 0 & -0.4073 & 70 & -0.40705803061326 & $2.2 \cdot 10^{-19}$ & -0.40705803061340 \\
0.01 & 2 & 1 & -0.1154 & 167 & -0.11524522409056 & $1.1 \cdot 10^{-19}$ & -0.11524522409056 \\
0.01 & 3 & 1 & -0.0468 & 188 & -0.04615310482916 & $8.2 \cdot 10^{-20}$ & -0.04615310482916 \\
0.01 & 3 & 2 & -0.0463 & 176 & -0.04606145416066 & $5.4 \cdot 10^{-20}$ & -0.04606145416065 \\
0.01 & 9 & 0 & -0.00111 & 350 & -0.00058524761250 & $7.2 \cdot 10^{-21}$ & -0.00058524761250 \\
0.01 & 9 & 1 & -0.00106 & 1034 & -0.00056650762617 & $2.5 \cdot 10^{-21}$ & -0.0005665076261 \\
\hline
\end{tabular}

Table 3: Example (21)-(22): Comparison of the eigenvalues of the Yukawa potential for several values of $\alpha$ reported in [26]. For the computations we prescribed $t o l_{a}=10^{-15}$ and tol $l_{r}=10^{-10}$.

The next example we discuss is the Schrödinger equation with the Hulthén potential, which corresponds to $(21)-(22)$ for $V(r)=-\frac{\alpha \mathrm{e}^{-\alpha r}}{1-\mathrm{e}^{-\alpha r}}$ [26]. Table 4 gives a comparison of our results with those from [26], where the displayed data corresponds with that in Table 3. According to [4], for $l=0$ exact eigenvalues can be determined analytically for the Hulthén potential. These are given by

$$
\lambda_{n}^{\text {exact }}=-\frac{1}{2}\left(\frac{1}{n}-\frac{n \alpha}{2}\right)^{2} .
$$

The resulting value corresponding to the first row in Table 4 is $\lambda=-0.49900050000000$. The error resulting with our numerical method is approximately equal to $10^{-13}$ [28]. 


\begin{tabular}{|r|c|c|r|r|r|r|r|}
\hline$\alpha$ & $n$ & $l$ & \multicolumn{1}{|c|}{$\lambda^{(0)}(\mathrm{MM})$} & $N$ & \multicolumn{1}{c|}{ bvpsuite } & errest $_{\lambda}$ & {$[26]$} \\
\hline 0.002 & 1 & 0 & -0.4993 & 67 & -0.49900049999985 & $8.7 \cdot 10^{-18}$ & -0.49900050000000 \\
0.15 & 3 & 2 & -0.0015 & 235 & -0.00139659246573 & $1.9 \cdot 10^{-20}$ & -0.00139659246573 \\
0.02 & 8 & 1 & -0.0012 & 1441 & -0.0009868327076 & $2.6 \cdot 10^{-21}$ & -0.0009868327076 \\
\hline
\end{tabular}

Table 4: Example: Comparison of the eigenvalues of the Hulthén potential for several values of $\alpha$ reported in [26]. For the computations we prescribed $t o l_{a}=10^{-15}$ and $t o l_{r}=10^{-10}$.

Finally, we give numerical results for a Schrödinger equation with matrix-valued potential to demonstrate that systems of equations can be treated in a natural way by our code without any special handling. We discuss the hydrogen atom in a dc-electric field in $x_{3}$-direction, the Stark effect [19, 27], which is given by the following PDE-operator

$$
H(\mathcal{E})=-\frac{1}{2} \triangle-\frac{1}{r}-\mathcal{E} f_{b}(r) x_{3}=-\frac{1}{2} \triangle-\frac{1}{r}-\sqrt{\frac{4 \pi}{3}} \mathcal{E} f_{b}(r) r Y_{10}(x),
$$

where $x=\left(x_{1}, x_{2}, x_{3}\right)$. The function $f_{b}(r)$ makes the perturbation decay to zero for large $r$ and could be chosen as $f_{b}(r):=e^{-b r^{2}}, b \geq 0$. The potential is no longer spherically symmetric and therefore no decoupled radial ODEs are obtained. Standard expansion into spherical harmonics $Y_{\ell m}$ and truncation at $l_{\max }=1$ lead to two decoupled ODEs and the ODE system

$$
\begin{aligned}
& {\left[-\frac{1}{2} \frac{d^{2}}{d r^{2}}+\left(\begin{array}{cc}
-\frac{1}{r} & -\frac{\mathcal{E}}{\sqrt{3}} r f_{b}(r) \\
\frac{\mathcal{E}}{\sqrt{3}} r f_{b}(r) & -\frac{1}{r}+\frac{1}{r^{2}}
\end{array}\right)\right] y(r)=\lambda y(r), \quad r \in(0, \infty),} \\
& y(0)=0, y(\infty)=0 .
\end{aligned}
$$

It turns out that our approach works equally well for $b=0$ which we will henceforth use. For this problem, the eigenvalues can be determined approximately by time-independent perturbation theory in the parameter $\mathcal{E}$, the lowest eigenvalues split up in 2nd order perturbation theory [19]:

\begin{tabular}{|r|r|l|}
\hline$n$ & $m$ & $\lambda=\lambda(\mathcal{E})$ \\
\hline 1 & 0 & $-\frac{1}{2}-\frac{9}{4} \mathcal{E}^{2}$ \\
2 & 0 & $-\frac{1}{8}+3 \mathcal{E}-84 \mathcal{E}^{2}$ \\
2 & 0 & $-\frac{1}{8}-3 \mathcal{E}-84 \mathcal{E}^{2}$ \\
2 & -1 & $-\frac{1}{8}-78 \mathcal{E}^{2}$ \\
2 & 1 & $-\frac{1}{8}-78 \mathcal{E}^{2}$ \\
\hline
\end{tabular}

Table 5: Perturbation analysis of the Stark effect.

Table 6 shows our numerical results for $\mathcal{E}=10^{-4}$ as compared to the values determined by perturbation theory. 


\begin{tabular}{|c|r|c|c|c|c|}
\hline$n$ & $\lambda^{(0)}(\mathrm{MM})$ & $N$ & bvpsuite & errest $_{\lambda}$ & $\lambda_{\text {per }}$ \\
\hline 1 & -0.5001 & 100 & -0.500000022499859 & $4.4 \cdot 10^{-19}$ & -0.500000022500000 \\
2 & -0.1253 & 163 & -0.125300320116262 & $2.2 \cdot 10^{-19}$ & -0.125300840000000 \\
3 & -0.1247 & 163 & -0.124700319903047 & $1.1 \cdot 10^{-19}$ & -0.124700840000000 \\
\hline
\end{tabular}

Table 6: Example (25): The computed eigenvalues $\lambda$ for $\mathcal{E}=10^{-4}$ and $b=0$ as compared to the results from perturbation theory. The tolerances were set to $t_{o} l_{a}=10^{-15}$ and $t_{0} l_{r}=$ $10^{-10}$.

\section{Conclusions}

We have presented an alternative method for the solution of eigenvalue problems for singular ODEs which is very well suited for the radial Schrödinger equation. Our approach is based on a routine to determine rough approximations to several of the eigenvalues and eigenfunctions simultaneously, and subsequent refinement by an adaptive collocation method which additionally yields reliable error estimates. This method can be efficiently applied to a more general class of singular boundary value problems, but can also compete with methods especially adapted to the linear second order radial Schrödinger equation. After briefly reviewing the key computational steps we apply our method to a few standard problems of physical interest with encouraging results. Especially a radial coupled system of ODEs can be treated without further adaptation. Therefore we can directly study the dc-Stark effect. A comparison with known results from perturbation theory and results from the literature demonstrate the precision of our approach. Since standard core state solvers in electronic structure theory still use only the spherical part of the potential, our method can be used to extend such routines.

\section{References}

[1] W. Auzinger, E. Karner, O. Koch, E. Weinmüller, Collocation methods for the solution of eigenvalue problems for singular ordinary differential equations, Opuscula Math. 26 (2) (2006) 229-241.

[2] W. Auzinger, O. Koch, E. Weinmüller, Collocation methods for boundary value problems with an essential singularity, in: I. Lirkov, S. Margenov, J. Wasniewski, P. Yalamov (eds.), Large-Scale Scientific Computing, vol. 2907 of Lecture Notes in Computer Science, Springer Verlag, 2004.

[3] P. Bailey, W. Everitt, A. Zettl, Algorithm 810: The SLEIGN2 Sturm-Liouville code, ACM Trans. Math. Software 27 (2) (2001) 143-192.

[4] O. Bayrak, I. Boztosun, Analytical solutions of the Hulthén and the Morse potentials by using the Asymptotic Iteration Method, J. Mol. Structure: THEOCHEM 802 (2007) $17-21$. 
[5] C. J. Budd, O. Koch, E. Weinmüller, Computation of self-similar solution profiles for the nonlinear Schrödinger equation, Computing 77 (2006) 335-346.

[6] C. J. Budd, O. Koch, E. Weinmüller, From nonlinear PDEs to singular ODEs, Appl. Numer. Math. 56 (2006) 413-422.

[7] J. Cooley, An improved eigenvalue corrector formula for solving schroedinger equation for central fields, Math. Comp. 15 (1961) 363-374.

[8] J. P. Desclaux, Hartree-Fock Slater self consistent field calculations, Comput. Phys. Commun. 1 (3) (1970) $216-222$.

[9] J. P. Desclaux, A multiconfiguration relativistic DIRAC-FOCK program, Comput. Phys. Commun. 9 (1) (1975) $31-45$.

[10] B. Drittler, M. Weinert, R. Zeller, P. H. Dederichs, Electric-field gradients in dilute Cu alloys: The role of the Cu d electrons, Phys. Rev. B 42 (15) (1990) 9336-9339.

[11] D. Hartree, The calculation of atomic structures, John Wiley, 1957.

[12] F. d. Hoog, R. Weiss, Difference methods for boundary value problems with a singularity of the first kind, SIAM J. Numer. Anal. 13 (1976) 775-813.

[13] F. d. Hoog, R. Weiss, Collocation methods for singular boundary value problems, SIAM J. Numer. Anal. 15 (1978) 198-217.

[14] F. d. Hoog, R. Weiss, The numerical solution of boundary value problems with an essential singularity, SIAM J. Numer. Anal. 16 (1979) 637-669.

[15] H. Keller, On the accuracy of finite difference approximations to the eigenvalues of differential and integral operators, Numer. Math. 7 (1965) 412-419.

[16] G. Kitzhofer, O. Koch, P. Lima, E. Weinmüller, Efficient numerical solution of the density profile equation in hydrodynamics, J. Sci. Comput. 32 (2007) 411-424.

[17] G. Kitzhofer, O. Koch, E. Weinmüller, The new MATLAB code BVPSUITE for the solution of implicit boundary value problems, submitted to JNAIAM J. Numer. Anal. Indust. Appl. Math. (2009).

[18] H.-O. Kreiss, Difference approximations for boundary and eigenvalue problems for ordinary differential equations, Math. Comp. 26 (119) (1972) 605-624.

[19] L. D. Landau, L. M. Lifshitz, Quantum Mechanics: Non-Relativistic Theory, vol. 3, Butterworth-Heinemann, Oxford, U.K., 1981.

[20] V. Ledoux, Study of Special Algorithms for Solving Sturm-Liouville and Schrödinger Equations, Ph. D. Thesis, Department of Applied Mathematics and Computer Science, Ghent University (2007). 
[21] V. Ledoux, M. Van Daele, G. Vanden Berghe, A numerical procedure to solve the multichannel Schrödinger eigenvalue problem, Comput. Phys. Commun. 176 (2006) 191199.

[22] M. Marletta, J. Pryce, A new multipurpose software package for Schrödinger and SturmLiouville computations, Comput. Phys. Commun. 62 (1991) 42-52.

[23] R. Martin, Electronic Structure: Basic Theory and Practical Methods, Cambridge University Press, 2008.

[24] S. Pruess, C. Fulton, Mathematical software for Sturm-Liouville problems, ACM Trans. Math. Software 19 (3) (1900) 360-376.

[25] J. Pryce, Numerical Solution of Sturm-Liouville Problems, Oxford University Press, New York, 1993.

[26] A. Roy, The generalized pseudospectral approach to the bound states of the Hulthn and the Yukawa potentials, PRAMANA - J. Phys. 65 (2005) 1-15.

[27] F. Schwabl, Quantenmechanik, Springer Verlag, Berlin; Heidelberg, 1993.

[28] C. Simon, Numerical solution of singular eigenvalue value problems for systems of odes with a focus on problems posed on semi-infinite intervals, Master thesis, Vienna Univ. of Technology, Vienna, Austria (2009).

[29] T. Simos, Explicit exponentially fitted methods for the numerical solution of the Schrödinger equation, Appl. Math. Comput. 98 (2-3) (1999) 185 - 198.

[30] J. Slater, Quantum Theory of Molecules and Solids, Vol.1-Vol.4, McGraw-Hill, New York, 1963.

[31] G. Teschl, Mathematical Methods in Quantum Physics, Graduate Studies in Mathematics, Amer. Math. Soc., Providence, 2009.

[32] J. Zabloudil, R. Hammerling, L. Szunyogh, P. Weinberger, Bulk and surface properties of metals by full-charge-density screened Korringa-Kohn-Rostoker calculations, Phys. Rev. B 73 (11) (2006) 115410. 